\title{
Research on Architecture of Military Information Network Based on SDN
}

\author{
Wenxin Qiao ${ }^{1,}$, , Zhen $\mathrm{Han}^{2}$ and Liu Bo ${ }^{3}$ \\ ${ }^{1}$ Information Engineering Department Mechanical Engineering CollegeShijiazhuang, China \\ ${ }^{2}$ China Aerodynamics Research and Development CenterMianyang, China \\ ${ }^{3}$ North Automatic Control Technology Institute, Taiyuan, China \\ aqiaowenxin1992@foxmail.com
}

Keywords: SDN, military information network, operational efficiency, security protection.

\begin{abstract}
A new scheme of military information network architecture was proposed based on the characteristics of SDN technology and military information network aiming at the new requirement of military information network. The management application layer is designed mainly from the security control center and the cloud data management center. Higher operational efficiency and dynamic security protection ability could be achieved through this model. The research of this paper may establish foundation for the construction of military information network in the future.
\end{abstract}

\section{I . Introduction}

The battlefield information has become the key to decide the outcome of a war in the era of information warfare. Military information network is the bottom information network to carry on the battlefield communication. The advance military information network is the key to realize the efficient and reliable information transmission. With the continuous development of information technology, the battlefield data information is explosive growth, which puts forward a new challenge to the traditional military information network.

Virtualization ${ }^{[1]}$ and software defined network $(\mathrm{SDN})^{[2]}$, as the research hotspot in the current information network, have considerable research prospects. They provide new ideas and methods to break the limitations of traditional networks. At present, there are a lot of cases based on virtualization and SDN to build a new type of information ${ }^{[3][4][5]}$. With the development of military information network, virtualization and SDN will be used in this area. This paper will study the architecture of military information network based on SDN. The detailed construction scheme is prosed and will provide a reference for the construction of new military network

\section{THE BASIC THEORY OF KNOWLEDGE}

\section{A. Demand Analysis of Military Information Network}

Military information network is mainly to realize the information flow processing in the military communication environment including the acquisition and transmission of the information flow, which is the basis of military communication. Task and security are the mainly demand of military information network in the new period.

In the aspect of task demand, it is one of the key factors to build a new military information network how to satisfy the efficient processing of multi node and multi service, and improve the efficiency of network management. In the terms of security requirements, it is another key consideration how to cope with the dynamic and varied types of network soft and hard attack in order to improve the security of network. 


\section{III . THE CONSTRUCTION SCHEME of MILITARY INFORMATION NETWORK ARCHITECTURE}

Due to the special nature of military information network, it needs to consider two aspects of task and security requirements in the construction of military information network. This section describes the construction of military information network architecture based on SDN technology.

\section{A. Overall Construction Scheme}

Virtualization technology can build a virtual computer and network resources on the basis of limited hardware resources which can flexibly achieve the required functions with a high utilization rate of resources. SDN is a breakthrough of the traditional network architecture. A relatively independent control plane and forwarding plane are formed in which the data in the traditional network control and forwarding solution of the coupled. The overall framework of the military information network is shown in figure 1 based on the above two points.

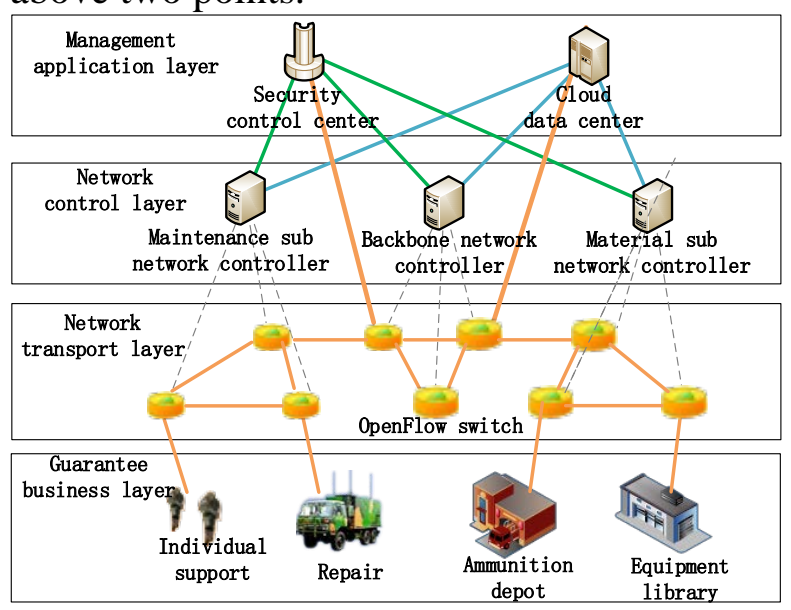

Figure1 Overall framework of military information network

The network system is divided into management application layer, network control layer, Network transmission layer and guarantee business layer according to the basic framework of application layer, control layer and infrastructure layer in SDN combined with the demands in military information network. Not only the requirement of each layer task for convenient management and function expansion but also the safety of the structure for the military information network control can be achieved using the characteristics of SDN control and forwarding phase separation.

\section{B. Detailed Construction Scheme}

Management application layer is the core of military information network, which directly determines the quality of military information ${ }^{[6]}$. The detailed design of this section is mainly from the two parts of the security control center and the cloud data center to design the management application layer.

(1)Security control center

Security control center is the core part of the whole network security protection. The basic structure is shown in figure 2. 


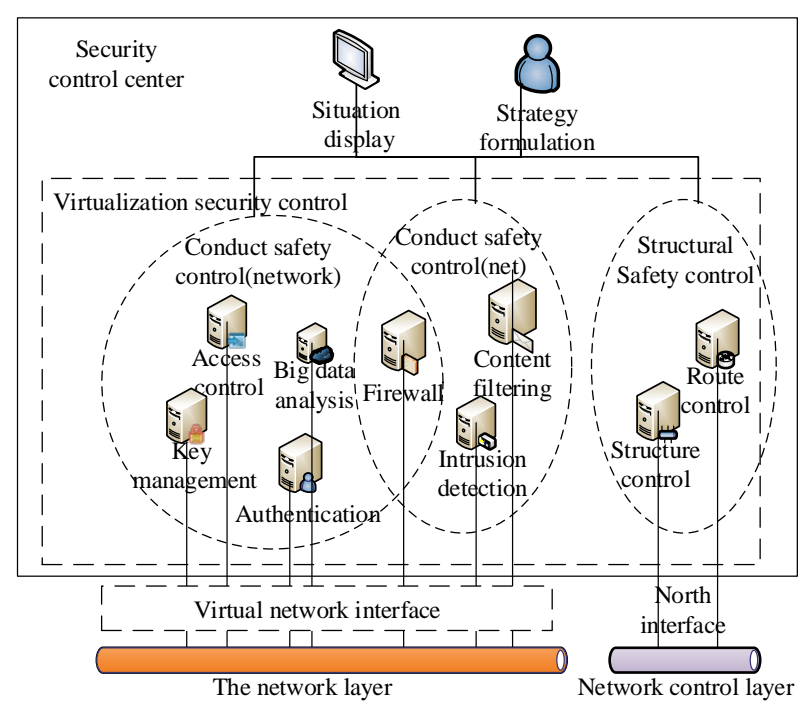

Figure 2 Basic structure of safety control center

Virtualization security control mechanism can realize the virtualization of security control. The basis of selecting a lot of security control mechanisms is the two aspects of behavior control and structure control in network theory. Behavior control is complex which is divided into external and internal network and has directly access to the network transport layer through the virtual network interface. Structure control is connected with the network control player via the north of SDN based on the characteristics of centralized management and control of logic topology by SDN. The safety control in the center of the virtual safety control is regarded either as the safety on network system in the upper application expansion which reflects the flexible network system programming or part of a network which can achieve dynamic security control of the entire network.

(2) Cloud data center

Cloud data center is the core of the entire network application services which provides computing, storage and network services for military information network. The basic structure is shown in figure 3.

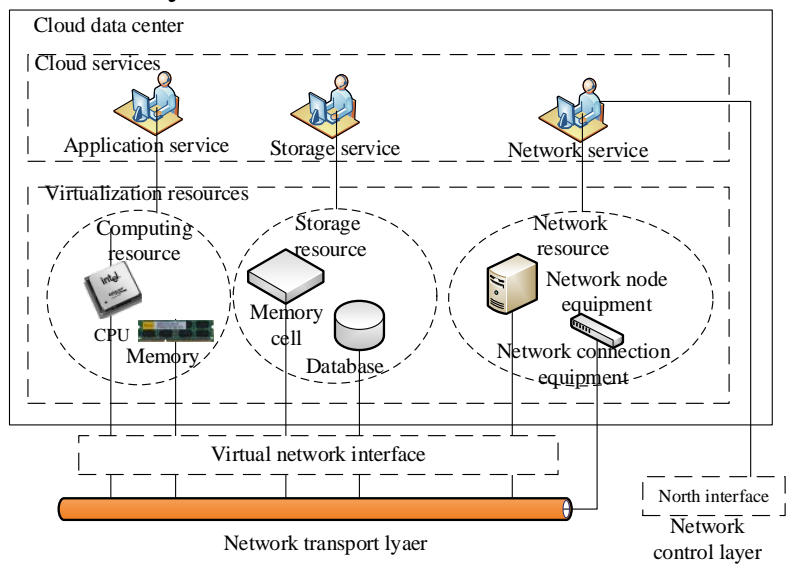

Figure 3 Basic structure of cloud data center

It is easily known that the cloud data center mainly provides three kinds of virtualization resources and corresponding cloud services for military information network. All kinds of virtual resources and services are based on virtual technology. The main computing resources includes CPU and memory which can provides computing resource management and all kinds of equipment support business in the calculation of the required processing etc. Storage resource is consist of unit and database which provides services including storage resource allocation, encryption storage, data backup and data migration etc. The network resources mainly include nodes and connections in the network providing 
network services consist of single backup, single machine migration, network fragmentation isolation, network load balancing and so on.

Virtualization resources has directly access to the network transport layer through the virtual network interface. The corresponding cloud also directly provide services for the network transport layer. The network service is also connect with the network control layer through the north interface in

order to realize some functions of the network ${ }^{[7]}$. Similar to the security control center, the cloud data center of the virtual resources and services can be seen as the upper application of the network system to expand the function services which reflects the flexibility of the network.

\section{Conclusion}

The construction scheme of a new military information network is studied based on virtualization and SDN combined with the mission requirements and security requirement of the military information network. The core idea of this paper is that the required network resources and security mechanism is constructed based on virtualization technology and the network slice and structure control is realized based on SDN. The network efficient operation and dynamic protection are meet by increasing the flexibility of the network system. SDN will be used in the military information network as the research focus of the current network construction. The construction scheme of this paper not only conform to the future development of the military information network, but also provides building ideas and reference for the new type military information network and has a certain reference value for the future information construction.

\section{References}

[1] CHOWDHUR N M, BOUTABA R. A survey of network virtualization [J]. Computer Networks, 2010, 54(5):862-876.

[2] Thomas D. Nadeau, Ken Gray. SDN: Software Defined Network [M].O’Reilly Media Inc,2013.

[3] ZHANG Hao, DONG Xiao-She, WANG En-Dong, etc. Reliability Architecture to Isolate the Driver Inside the VM [J]. Journal of software, 2014, 25(10):2235-2250.

[4] $\mathrm{Xu}$ Jie. Reliability, Maintainability and Survivability of the Weapon Equipment [J]. Marine Electric, 2012,32(3):45-46.

[5] ZUO Qing-Yun, CHEN Ming, ZHAO Guang-Song etc. Research on OpenFlow-Based SDN Technologies [J]. Journal of Software, 2013, 24 (5):1078-1097.

[6] DAI Bin, WANG Hang-yuan, XU Guang, YANG Jun. Opportunities and threats coexist in SDN security [J]. Application Research of Computers, 2014, 31(8):2254-2262.

[7] GUI Jing-Song, GUO Chi, CHEN Long etc. Establishing Process-Level Defense-in-Depth Framework for software Defined Network [J]. Journal of Software, 2013, 24 (5):1078-1097. 